\title{
Quality of Service Analysis of Ethernet Network Based on Packet Size
}

\author{
Nazrul Islam1, Chayan Chandra Bawn', Jahid Hasan', Asma Islam Swapna1, \\ Md. Syfur Rahman ${ }^{2 *}$ \\ ${ }^{1}$ Department of Information and Communication Technology, Mawlana Bhashani Science and Technology \\ University, Santosh, Bangladesh \\ ${ }^{2}$ Education Management Information System (EMIS) Cell, Directorate of Secondary and Higher Education, \\ Ministry of Education, Dhaka, Bangladesh \\ Email: nazrul.islam@mbstu.ac.bd, chayanbawn@gmail.com, jahidhasan.ict@gmail.com, \\ asma0swapna@gmail.com, "syfur022@gmail.com
}

Received 22 January 2016; accepted 20 March 2016; published 7 April 2016

Copyright (C) 2016 by authors and Scientific Research Publishing Inc.

This work is licensed under the Creative Commons Attribution International License (CC BY).

http://creativecommons.org/licenses/by/4.0/

(c) (i) Open Access

\begin{abstract}
Ethernet network, standardized by IEEE 802.3, is vastly installed in Local Area Network (LAN) for cheaper cost and reliability. With the emergence of cost effective and enhanced user experience needs, the Quality of Service (QoS) of the underlying Ethernet network has become a major issue. A network must provide predictable, reliable and guaranteed services. The required QoS on the network is achieved through managing the end-to-end delay, throughput, jitter, transmission rate and many other network performance parameters. The paper investigates QoS parameters based on packet size to analyze the network performance. Segmentation in packet size larger than 1500 bytes, Maximum Transmission Unit (MTU) of Ethernet, is used to divide the large data into small packets. A simulation process under Riverbed modeler 17.5 initiates several scenarios of the Ethernet network to depict the QoS metrics in the Ethernet topology. For analyzing the result from the simulation process, varying sized packets are considered. Hence, the network performance results in distinct throughput, end-to-end delay, packet loss ratio, bit error rate etc. for varying packet sizes.
\end{abstract}

Keywords

QoS, Ethernet Network, Performance Analysis, Packet Size, Segmentation

${ }^{*}$ Corresponding author.

How to cite this paper: Islam, N., Bawn, C.C., Hasan, J., Swapna, A.I. and Rahman, M.S. (2016) Quality of Service Analysis of Ethernet Network Based on Packet Size. Journal of Computer and Communications, 4, 63-72.

http://dx.doi.org/10.4236/jcc.2016.44006 


\section{Introduction}

Ethernet network is covered under IEEE 803.2 working group referring to the LAN family of network protocols. Since it is published as an official standard in 1985, several supplements have been defined to support additional network media and higher data rate compatibilities with the advancement of network technologies [1] [2]. Nowadays, Ethernet supports longer transmission distance up to $150 \mathrm{~km}$ which indicates the mass installation in an optical fiber network in most LAN environments. Ethernet network uses both packet and frame for the transmission using the physical and data link layer at the same time [3]. Ethernet stations communicate sending the data as packets where the Ether-Type field uses frames at the receiving station to select an appropriate protocol module by the operating system [4].

Performance is the key issue for the installation of Ethernet in any LAN environment. In the field of telecommunication QoS is defined through some network parameters such as delivery rate, throughput, delay, collision probability, bandwidth efficiency, packet loss ratio, bit error rate, queuing delay, and jitter [5]. Ethernet has MTU of 1500 bytes which is much higher and potential to degrade the performance of the network [6]. However, if the packet sizes increase in the transmission, congestion occurs and probability of packet drop increases. Segmenting the larger packets to divide into smaller ones avoids congestion and impacts on QoS on the overall Ethernet network. Packet size impacts on the queuing in the switch and router's buffer during the transmission process among the Ethernet stations. This varies the packet arrival and departure rate consequently the packet processing rate [7]. When the packet size exceeds the MTU, the end-to-end packet arrival/departure delay increases [7]. Segmentation requires time and impacts on the data rate and degrades the QoS of the network. Thus, larger, packet size leads to network congestion while smaller one is capable to lower the packet arrival and departure delay and decrease the bit error rate [8]. This study represents the analysis on QoS and network performance while segmentation of such larger packets in the Ethernet network is simulated.

Previously performed studies in [9] depict that lower data rate of application due to larger packet size lowers the energy efficiency of the network. Another study in [10] represents that optimal packet size improves performance of error prone channel of the wireless LAN. Carmo et al. in [2] presented that the prioritization of the traffic can affect the performance of the overall network. Hence, segmentation of the packet size for transmission of data between the Ethernet stations is capable to impact on overall network performance [11].

This study evaluates the QoS of an Ethernet network simulated on Riverbed modeler that depicts the impacts of varying packet size in the network QoS and performance parameters based on the previous research findings. The purpose of this segmentation process is to analyze the performance parameters with larger packet sizes than MTU and evaluate how that impacts the throughput, bit error, delay and thus the overall network QoS compared to the performance of smaller packet's transmission process.

According to the experiments and findings this paper is structured in the followings: Section 2 represents the methodology of the paper. Section 3 shows the design and implementation of the LAN based Ethernet Network. The detailed results and analysis of QoS parameters after segmentation among larger packets are presented in Section 4. Section 5 concludes with the set of observation and future direction of the research.

\section{Related Work}

Several research is performed in the field of network congestion control mechanism showing improved network performance for different packet sizes [12]. The author of [9] proved that, the lower of an application data rate, the lower its energy efficiency. That implies, if the data rate is low due to large packet size, then the network consumes more energy. Optimal packet size can have a significant improvement on energy efficiency under error prone channel on a wireless local area network [10]. Large propagation delays in underwater networks, rather than being harmful, lead to significant performance gains as compared to wireless networks with negligible propagation delays [8]. An important issue related to the network performance is congestion, which may occur in a network when the number of packets sent to the network is greater than the number of packets that the network can handle.

The network device that is used as intermediary routers and switches in a network have buffers where the packets wait in a queue before and after processing the service. Based on the packet arrival rate and the packet departure rate, which may be higher or lesser than the packet processing rate, the size of the input or the output queue may increase or decrease. This increase in queue size can be considered to lead network congestion [7]. Congestion impacts on network performance. Such congestion control evaluates the network performance 
through-put and network delay. Using Bandwidth prediction algorithm Muttukrishnan et al. implemented a Hybrid Local Area Network with improved QoS, robustness and effectiveness for both Wired and Wireless [13]. In the paper [14], timing analysis with end-to-end packet delivery is derived to observe the switched Ethernet Network performance. However, this study represents several performance parameters to study the QoS packet segmentating the larger packets while transmission.

\section{Methodology}

A literature review is performed in several application areas of Ethernet LAN and the Quality of Service of such a network to calculate the delay, throughput, packet loss ratio, bit error rate and queuing delay. The perspective research on the packet format for Ethernet network is performed to analyse the transmission process. The packet format is depicted in the Figure 1(a). A parallel study is performed on the segmentation process for the packets which are higher in size than MTU. Larger data packets than 1500 bytes will split into smaller packets called fragments to enable them transverse the network [15]. Based on the literature study a proper simulation tool is required to develop the network in the real time simulation.

Among several network simulation tools including OMNET++, Network Simulator-2 (NS-2), Network Simulator-3 (NS-3), Riverbed [16], and MATLAB qualitative analysis was performed in this study. Hence, Riverbed molder 17.5 was chosen for easy development environment and wide range of credence to perform such simulation [17]. The simulator was installed in a core i3 based workstation. Ethernet network, considered here has deployed the Fast-Ethernet link. The reason of considering fast Ethernet is its vast use in optical communication, laboratory atmosphere for higher range of speed up to $10 \mathrm{Gbit} / \mathrm{s}$ [18]. A testbed of Ethernet LAN is structured with 16 client stations, two hubs and a switch. In the topology each of the two hubs is connected with 8 client stations using a full duplex 10BASE_T link as a fast Ethernet link which speed is 10 Mbps. The topology is depicted in Figure 1(b).

\begin{tabular}{ccccccc}
\hline \multicolumn{7}{c}{ Ethernet Packet } \\
PREAMBLE & SYNCH & DATA ADDRESS & SOURCE ADDRESS & TYPE OR LENGTH & DATA & FCS \\
\hline \multirow{2}{*}{62 BYTES } & 2 BYTES & \multirow{2}{*}{ 6 BYTES } & 6 BYTES & 2 BYTES & $46-1500$ BYTES & 4 BYTES \\
\hline
\end{tabular}

(a)

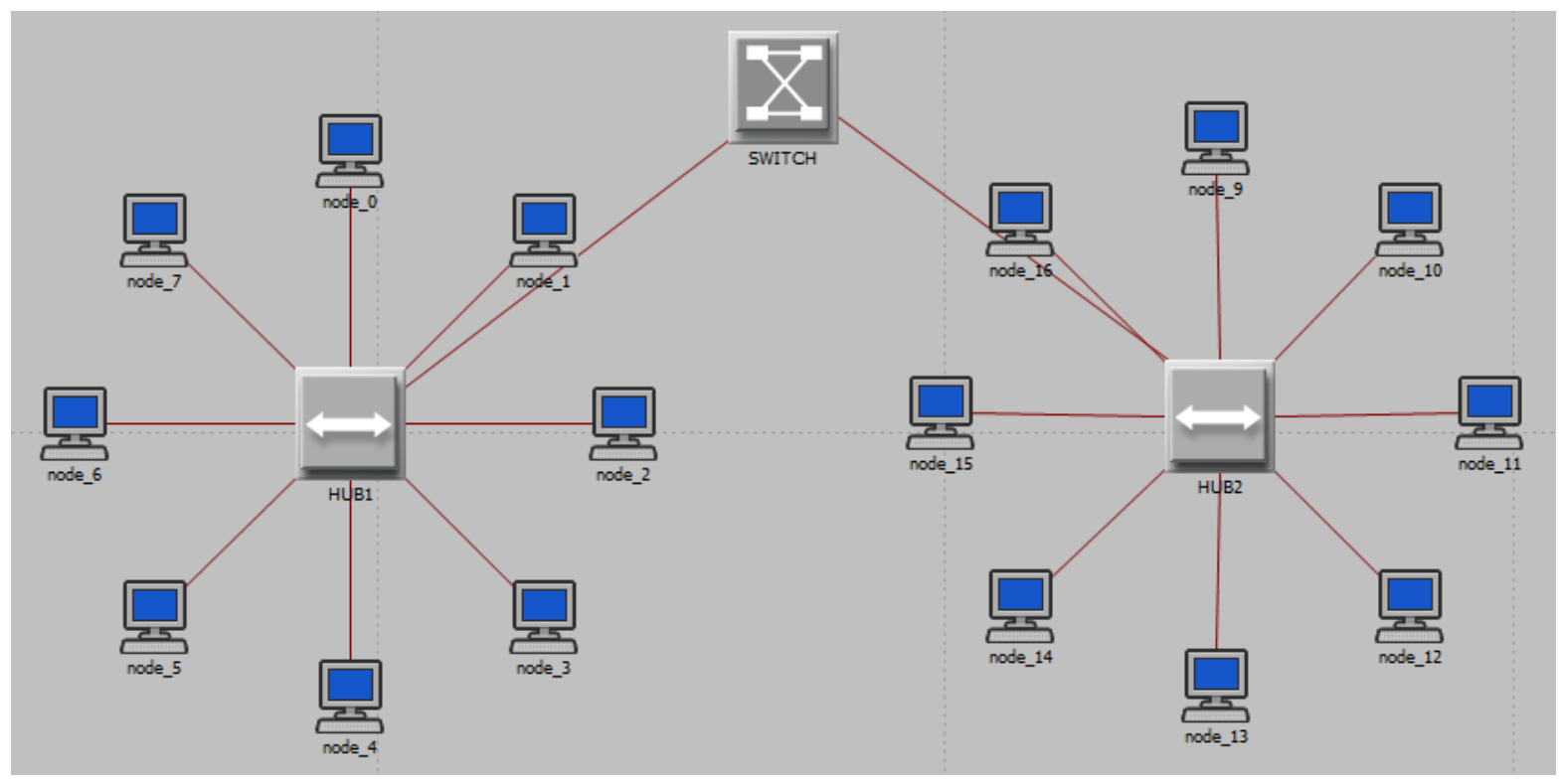

(b)

Figure 1. (a) Ethernet packet format; (b) Ethernet network. 
Through full duplex link among the hub and switch several packet transmits towards and from the clients. Larger packets with size greater than MTU are segmented into packet series called fragments. Without segmentation, larger packets fail to reach the destination on time with the required data and receiver sends the packet request. This proposed network segments the larger packets with 1500 Bytes and 1024 Bytes respectively. To analyze the network performance, each QoS parameters, including throughput, delay, packet loss ratio, queuing delay and bit error rate parameters, are considered as a simulation scenario. In this testbed, several scenarios with different sized packet transmission is performed and simulated in Riverbed about 300 seconds.

Finally, simulated data are collected carefully from Ethernet network testbed. At the end of the simulation the appropriate graphs are plotted to analyze each of the parameters. A careful study of the plots provides a quantitative measure of the effect of different network parameters in the Ethernet network.

\section{Design and Simulation}

The main focus of this thesis is to investigate the Quality of Service of an Ethernet network based on packet size. An experimental setup is configured and designed with 8 experimental scenarios. The Riverbed network simulator is used to observe the network behavior upon different packet sizes. The simulation results from Riverbed simulator are very close to real life scenarios and provide high level Ethernet network development features.

Ethernet LAN was designed for the low cost, flexibility in installation and decentralized IP packet transmissions among 265 hosts upto 3 Mbps capacity [19]. Today a Fast Ethernet type is used for high throughput host that can provide 10 Gbps full-duplex collision free link. In order to analyze the effect of packet size on QoS in Ethernet network, an experimental Ethernet LAN environment is established having 16 workstations, two hubs and a switch, illustrated in Figure 1(a). Each of the two hubs is connected with eight workstations by a full duplex link. The switch is connected with the hubs by a full duplex link. All the devices are connected though a link of bandwidth 10 Mbps. The client machine is configured using Microsoft Windows 7 and it is preferred as its one of the most popular and widely used operating systems [20]. We created 7 scenarios to carry out the experiment varying the packet sizes (128 Bytes, 256 Bytes, 512 Bytes, 1024 Bytes and 2048 Bytes). When a packet size exceeds maximum transmission unit of Ethernet network of 1518 Bytes we segmented the packet. Packet size for 8 different scenarios is given below Table 1 .

The traffic generation parameters and Ethernet parameters for all the scenarios are same. They are presented in Tables 2-4 in the following.

Table 1. Packet generation parameters for different scenarios.

\begin{tabular}{cccc}
\hline Scenario No. & Inter arrival time (seconds) & Packet size (Bytes) & Segmentation Size (Bytes) \\
\hline 1 & Constant $(0.03)$ & Constant (128) & No segmentation \\
2 & Constant $(0.03)$ & Constant (256) & No segmentation \\
3 & Constant $(0.03)$ & Constant (512) segmentation \\
4 & Constant $(0.03)$ & Constant (1024) & No segmentation \\
5 & Constant (0.03) & Constant (2048) & No segmentation \\
6 & Constant (0.03) & Constant (2048) & 1500 \\
7 & Constant (0.03) & Constant (2048) & 1024 \\
\hline
\end{tabular}

Table 2. Traffic generation parameters.

$\begin{array}{cc}\text { Start Time (Second) } & \text { Constant (5.0) } \\ \text { ON State Time (Second) } & \text { Constant (500) } \\ \text { OFF State Time (Second) } & \text { Constant (0) }\end{array}$

Table 3. Ethernet parameters.

\begin{tabular}{cc}
\hline Address & Auto Assigned \\
Frame Bursting & Enabled \\
Operational Mode & Full Duplex \\
Promiscuous Mode & Disabled \\
\hline
\end{tabular}


Table 4. Link parameters.

\begin{tabular}{cc}
\hline Propagation Speed & Speed of Light (in twisted pair) \\
\hline Delay & Distance Based \\
\hline
\end{tabular}

\section{Simulation Results}

This section offers competitive description and the analysis of the obtained discrete event simulation result. The result is based on the packet size in the Ethernet network observing its performance analysis. The graphs are generated using different Quality of service parameters (Packet loss ratio, Throughput, Queuing delay, Bit error rate, Delay etc.).

\subsection{Throughput}

Throughput is considered as one of the vital QoS parameters for networks [21]. Higher throughput is always expected. In general terms; throughput is the rate of production or the rate at which something can be processed. The simulation result shows the highest throughput with larger packet sizes near to 1024 Bytes (Figure 2).

\subsection{Delay}

The network delay specifies how long it takes for a bit of data to travel across the network from one node or endpoint to another. It is typically measured in multiples or fractions of seconds. Low network delay is essential for better network performance. Figure 3 illustrates the Ethernet network delay for different packet size. We can see that delay is proportion to packet size. This means small packet size, low delay and higher packet Sizes produce higher delay.

\subsection{Packet Loss Ratio}

The QoS of an Ethernet largely depends on the network performance parameter packet loss ratio. Large packet loss ratio degrades a network performance. As large number of packet losses increasing the retransmission, which fall down the network performance. Packet loss ratio is described as the ratio of lost packet and the total packet per unit of time. Number of lost packet/(Number of lost packet + Number of packets received successfully). Low packet loss ratio is always expected for better QoS performance. Figure 4 presents the simulation result of packet loss ratio for 128, 256, 512 and 1024 Bytes packets.

\subsection{Queuing Delay}

Queuing delay represents the queuing delay that packets from the network layer incur at the MAC queue until it can be executed [22]. It is the main component of overall network delay. Model has huge queuing delay for large packet size. Less queuing delay is expected to avoid performance degradation of an Ethernet network.

\subsection{Bit Error Per Packet}

This QoS parameter represents the average number of bit errors ina packet sent over a channel [23]. Bit error per packet plays a vital role for QoS of an Ethernet network. The Less bit error per packet improves the network performance. Hence, less bit error per packet is expected. Figure 5 illustrates the result of bit error per packet based on packet size. Figure 6 shows that in 128 Bytes packet size, bit error per packet are 0 and bit errors per packet increases with the increase of packet size.

\subsection{Traffic Forwards}

Amount of traffic forwarded per unit of time. Figure 7 shows that when packet size exceeds the MTU then no traffic, forwards form switches which means all packets are dropped.

\subsection{Throughput for Segmented Packets}

In general terms; throughput is the rate of production or the rate at which something can be processed. When 
Throughput (bits/sec)

—LAN_pack-pktSize128-DES-1. throughput (bits/sec)

LAN_pack-pktSize256-DES-1. throughput (bits/sec)

LAN_pack-pktSize512-DES-1. throughput (bits/sec)

LAN_pack-pktSize1024-DES-1. throughput (bits/sec)

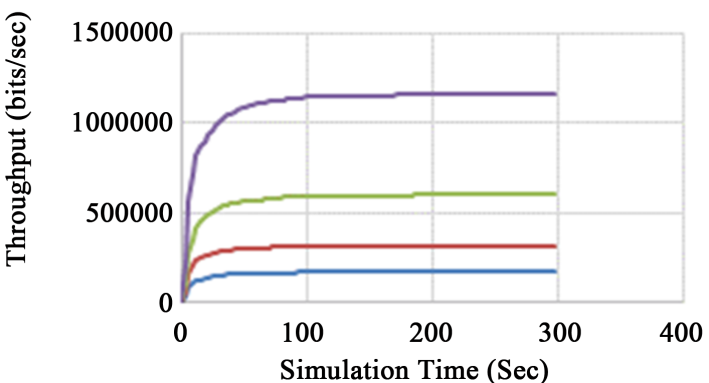

Figure 2. Throughput (bits/sec) at switch.

$$
\text { Delay (sec) }
$$

— LAN_pack-pktSize128-DES-1: EthernetDelay (sec)

LAN_pack-pktSize256-DES-1: EthernetDelay (sec)

LAN_pack-pktSize512-DES-1: EthernetDelay (sec)

— LAN_pack-pktSize1024-DES-1: EthernetDelay (sec)

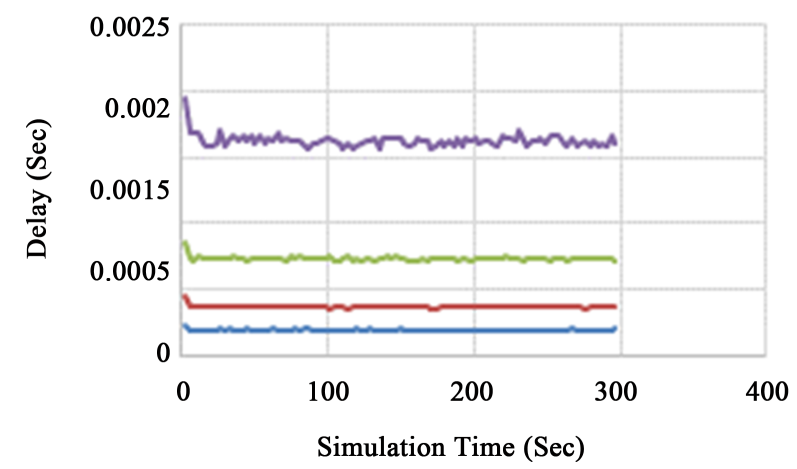

Figure 3. Network delay (sec) for 128, 256, 512 and 1024 Bytes packet size.

Packet Loss Ratio

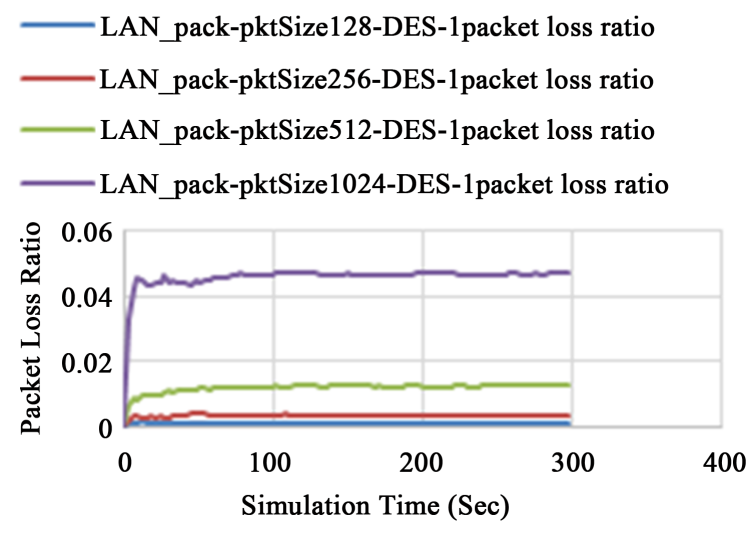

Figure 4. Packet loss ratio for 128, 256, 512 and 1024 Bytes packet size. 
used in the context of communication networks, such as Ethernet, throughput or network throughput is the rate of successful message delivery over a communication channel. Throughput is considered as one of the most important QoS parameters for networks. Higher throughput is always expected. Figure 8 illustrates the throughput when packet size exceeds MTU and hence segmentation is needed [24]. For 2048 Byte packet size two different segmentation sizes (1500 Bytes and 1024 Bytes) were used for Scenario 6 and Scenario 7 respectively. The simulation result shows that throughput for segmentation, size The simulation result shows the higher throughput with larger packet sizes near to 1024 bytes. Bytes and 1024 Bytes is almost same for the Ethernet network.

\section{Bit Error Per Packet}
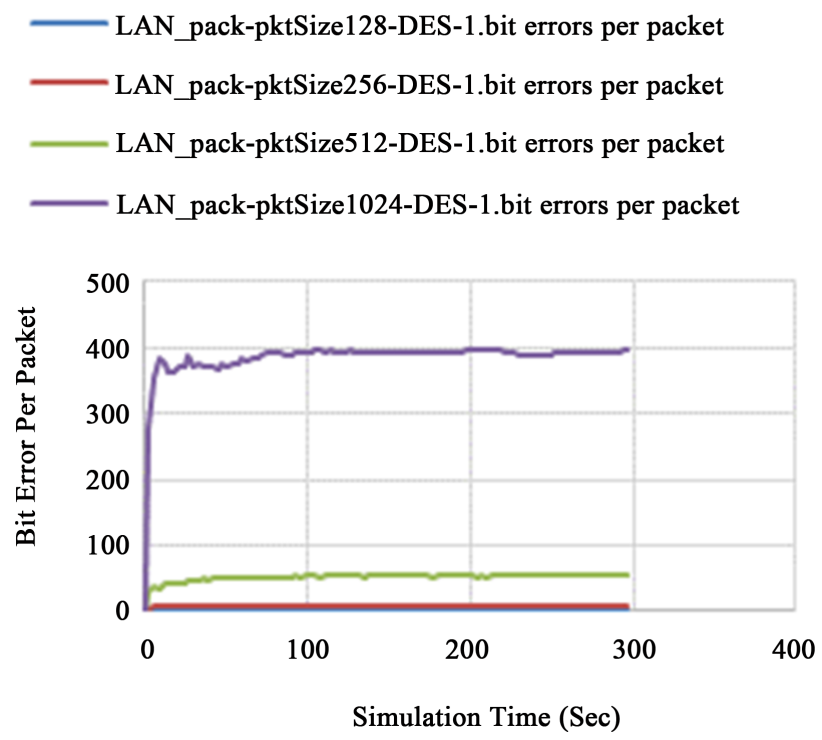

Figure 5. Bit error per packet for 128, 256, 512 and 1024 Byte packet size.

Queuing delay (sec)

LAN_pack-pktSize128-DES-1.queuing delay (sec)

LAN_pack-pktSize256-DES-1.queuing delay (sec)

LAN_pack-pktSize512-DES-1.queuing delay (sec)

LAN_pack-pktSize1024-DES-1.queuing delay (sec)

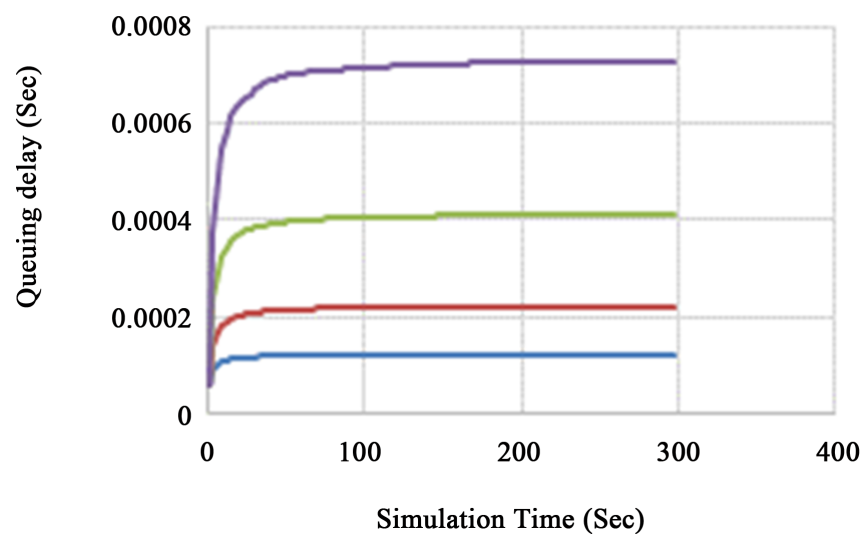

Figure 6. Queuing delay (sec) for 128, 256, 512 and 1024 Bytes packet size. 
Traffic Forwarded (bits/sec)

LAN_pack-pktSize2048_NO_Segmentation-DES-

1. Traffic Frowarded (bits/sec)

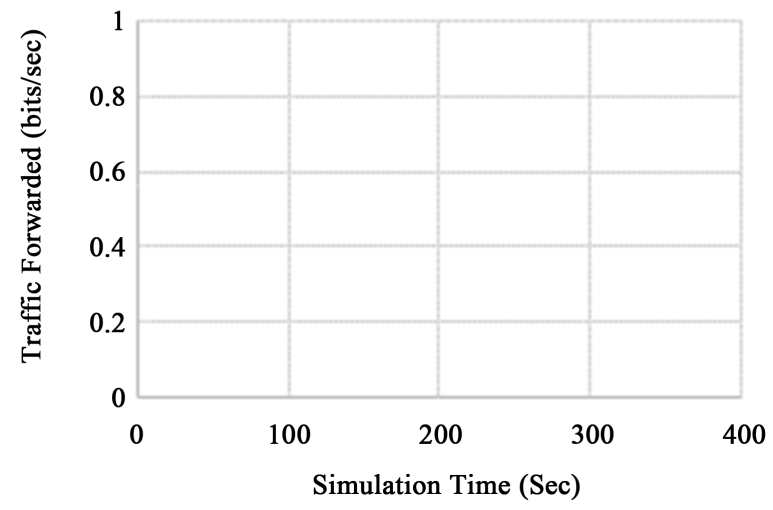

Figure 7. Traffic forwarded (bits/sec) when packet size exceeds MTU.

Throughput (bits/sec)

—LAN_pack-pktSize2048-DES-1.throughput (bits/sec)

—LAN_pack-pktSize2048seg1024-DES-1.throughput (bits/sec)

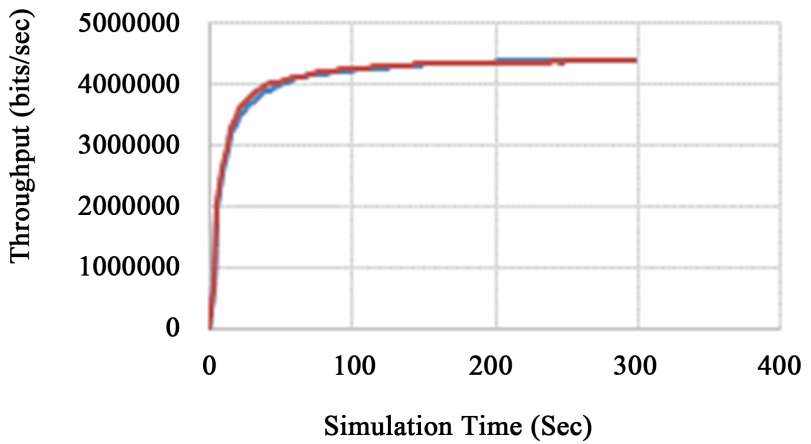

Figure 8. Throughput (bits/sec) for 1024 and 1500 Bytes segmentation size when packet size is 2048 Bytes.

\section{Conclusions}

The simulation presents a set of observations regarding impact of different packet sizes on the QoS in Ethernet local area network. Several QoS parameters came out with distinctive increase or decrease in performance. Segmentation is applied in the larger packet size of the Ethernet network. The simulation result represents that, for larger size packets than MTU of the network, throughput increases in a large amount implying better QoS for packet transmitted within the network. Meanwhile, for the larger packets no traffic forwarded from the switch. End-to-end delay increases exponentially for larger packets which drastically increases the queueing delay, packet loss ratio and error rate as well. This highly indicates that higher packet size gives inefficient QoS and less performance of the Ethernet network than the smaller packet's. Though there is a slightly better performance in highly increased throughput, however, the overall network performance degrades as most of the QoS parameters decreases for larger packet sizes than MTU in this testbed.

The future aspects of this performance fluctuation and findings can give a downbeat view on the QoS of the wireless environment for varying packet sizes. Segmentation of higher packet size can be a promising area to analyze the vastly used ZigBee network. Effect of packet size of Mobility network and VoIP application can be analyzed because the demand for such application is largely increasing day by day. 


\section{References}

[1] Beyranvand, H., Martin, L., Martin, M. and Salehi, J.A. (2015) FiWi Enhanced LTE-A HetNets with Unreliable Fiber Backhaul Sharing and WiFi Offloading. IEEE Conference on Computer Communications (INFOCOM), Kowloon, April 26 2015-May 1 2015, 1275-1283. http://dx.doi.org/10.1109/INFOCOM.2015.7218503

[2] Carmo, M., Sá Silva, J., Monteiro, E., Simões, P. and Boavida, F. (2005) Ethernet QoS Modeling in Emerging Scenarios. Proceedings of 3rd International Workshop on Internet Performance, Simulation, Monitoring and Measurement (IPS-MoMe 2005), Warsaw, 14-15 March 2005, 90-96.

[3] Salsano, S., Detti, A., Cancellieri, M., Pomposini, M. and Blefari-Melazzi, N. (2012) Transport-Layer Issues in Information Centric Networks. Proceedings of the Second Edition of the ICN Workshop on Information-Centric Networking, Helsinki, 17 August 2012, 19-24. http://dx.doi.org/10.1145/2342488.2342493

[4] Li, Y., Qi, X., Keally, M., Ren, Z., Zhou, G., Xiao, D. and Deng, S. (2013) Communication Energy Modeling and Optimization through Joint Packet Size Analysis of BSN and WiFi Networks. IEEE Transactions on Parallel and Distributed Systems, 24, 1741-1751. http://dx.doi.org/10.1109/TPDS.2012.264

[5] Braun, T., Diaz, M., Gabeiras, J.E. and Staub, T. (2008) End-to-End Quality of Service over Heterogeneous Networks. Springer Science \& Business Media.

[6] Das, R., Jain, V., Krishnan, U. and Venkatsubra, V. (2013) Efficient Path Maximum Transmission Unit Information Discovery and Storage. U.S. Patent No. 8,422,501.

[7] Gao, Y., Dai, L. and Hei, X. (2015) Throughput Optimization of Non-Real-Time Flows with Delay Guarantee of RealTime Flows in WLANs. IEEE International Conference on Communications (ICC), London, 8-12 June 2015, 15411546.

[8] Chitre, M., Motani, M. and Shahabudeen, S. (2012) Throughput of Networks with Large Propagation Delays. IEEE Journal of Oceanic Engineering, 37, 645-658. http://dx.doi.org/10.1109/JOE.2012.2203060

[9] Wang, X.D., Yin, J. and Agrawal, D.P. (2005) Effects of Contention Window and Packet Size on the Energy Efficiency of Wireless Local Area Network. IEEE Wireless Communications and Networking Conference, 1, 94-99.

[10] Tauber, M., Bhatti, S.N. and Yu, Y. (2011) Application Level Energy and Performance Measurements in a Wireless LAN. IEEE/ACM International Conference on Green Computing and Communications (GreenCom), Sichuan, 4-5 August 2011, 100-109. http://dx.doi.org/10.1109/greencom.2011.26

[11] Hussain, I., Ahmed, Z.I., Saikia, D.K. and Sarma, N. (2015) A QoS-Aware Dynamic Bandwidth Allocation Scheme for Multi-Hop WiFi-Based Long Distance Networks. EURASIP Journal on Wireless Communications and Networking, 1, 1-18. http://dx.doi.org/10.1186/s13638-015-0352-z

[12] Srikant, R. (2012) The Mathematics of Internet Congestion Control. Springer Science \& Business Media, Berlin, Heidelberg.

[13] Köhnen, C., Überall, C., Rajarajan, M., Jäger, R. and Rakočević, V. (2015) Autonomous QoS Management and Policing in Unmanaged Local Area Networks. Journal of Computer Networks and Communications, 2015, 1-24.

[14] Hassani, V., Talebi, H.A., Shafiee, M. and Taheri, H. (2007) Timing Analysis and Response Time of End to End Packet Delivery in Switched Ethernet Network. European Control Conference (ECC), Kos, 2-5 July 2007, 31-37.

[15] Yazid, M., Bouallouche-Medjkoune, L., Aïssani, D. and Ziane-Khodja, L. (2013) Analytical Analysis of Applying Packet Fragmentation Mechanism on IEEE 802.11b DCF Network in Non Ideal Channel with Infinite Load Conditions. Wireless Networks, 20, 917-934. http://dx.doi.org/10.1007/s11276-013-0653-2

[16] Modeler (2015) Riverbed Technology. http://www.riverbed.com

[17] Lu, Z. and Yang, H.J. (2012) Unlocking the Power of OPNET Modeler. Cambridge University Press, Cambridge.

[18] Ghassemlooy, Z., Le Minh, H., Rajbhandari, S., Perez, J. and Ijaz, M. (2012) Performance Analysis of Ethernet/ Fast-Ethernet Free Space Optical Communications in a Controlled Weak Turbulence Condition. Journal of Lightwave Technology, 30, 2188-2194. http://dx.doi.org/10.1109/JLT.2012.2194271

[19] Kiravuo, T., Sarela, M. and Manner, J. (2013) A Survey of Ethernet LAN Security. IEEE Communications Surveys \& Tutorials, 15, 1477-1491. http://dx.doi.org/10.1109/SURV.2012.121112.00190

[20] Hanay, Y.S., Li, W., Tessier, R. and Wolf, T. (2012) Saving Energy and Improving TCP Throughput with Rate Adaptation in Ethernet. IEEE International Conference on Communications (ICC), Ottawa, 10-15 June 2012, 1249-1254. http://dx.doi.org/10.1109/ICC.2012.6364655

[21] Queck, R. (2012) Analysis of Ethernet AVB for Automotive Networks Using Network Calculus. IEEE International Conference on Vehicular Electronics and Safety (ICVES), Istanbul, 24-27 July 2012, 61-67.

[22] Haigh, P.A., et al. (2012) Development of a Visible Light Communications System for Optical Wireless Local Area Networks. Computing, Communications and Applications Conference (ComComAp), Hong Kong, 11-13 January 2012, 351-355. http://dx.doi.org/10.1109/comcomap.2012.6154870 
[23] Valcarenghi, L., Van, D.P., Raponi, P.G., Castoldi, P., Campelo, D.R., Wong, S.W., Yen, S.H., Kazovsky, L.G. and Yamashita, S. (2012) Energy Efficiency in Passive Optical Networks: Where, When, and How? IEEE Network, 26, 61-68. http://dx.doi.org/10.1109/MNET.2012.6375895

[24] Hua, B., Hua, H.L. and Xiong, W. (2012) Changing Ethernet MTU Size on Demand with No Data Loss. U.S. Patent No. 8,214,535. 